OPEN

SUBJECT AREAS:

ASTROPHYSICAL

PLASMAS

PLANETARY SCIENCE

Received

10 March 2014

Accepted

12 May 2014

Published

9 June 2014

Correspondence and requests for materials should be addressed to

G.G. Ig.gregori1@ physics.ox.ac.uk)

\title{
Evidence for a glassy state in strongly driven carbon
}

\author{
C. R. D. Brown 1,2,3 , D. O. Gericke ${ }^{4}$, M. Cammarata ${ }^{5}$, B. I. Cho ${ }^{6,7,8}$, T. Döppner ${ }^{9}$, K. Engelhorn $^{6}$, E. Förster ${ }^{10}$, \\ C. Fortmann', D. Fritz ${ }^{5}$, E. Galtier ${ }^{11}$, S. H. Glenzer ${ }^{5}$, M. Harmand ${ }^{12}$, P. Heimann ${ }^{5}$, N. L. Kugland', \\ D. Q. Lamb ${ }^{13}$, H. J. Lee ${ }^{5}$, R. W. Lee ${ }^{6}$, H. Lemke ${ }^{5}$, M. Makita ${ }^{14}$, A. Moinard ${ }^{11}$, C. D. Murphy ${ }^{15}$, B. Nagler ${ }^{5}$, \\ P. Neumayer ${ }^{16}$, K.-U. Plagemann ${ }^{17}$, R. Redmer ${ }^{17}$, D. Riley ${ }^{14}$, F. B. Rosmej ${ }^{11}$, P. Sperling ${ }^{17}$, S. Toleikis ${ }^{12}$, \\ S. M. Vinko' , J. Vorberger ${ }^{4,18}$, S. White ${ }^{14}$, T. G. White' , K. Wünsch ${ }^{4}$, U. Zastrau ${ }^{5,19}$, D. Zhu ${ }^{5}$, \\ T. Tschentscher ${ }^{20} \&$ G. Gregori ${ }^{1,13}$
}

\begin{abstract}
'Department of Physics, Clarendon Laboratory, University of Oxford, Parks Road, Oxford OX1 3PU, UK, ${ }^{2}$ Plasma Physics Department, AWE plc., Aldermaston, Reading RG7 4PR, UK, ${ }^{3}$ Plasma Physics Group, Blackett Laboratory, Imperial College London, Prince Consort Road, London SW7 2AZ, UK, ${ }^{4}$ Centre for Fusion, Space and Astrophysics, Department of Physics, University of Warwick, Coventry CV4 7AL, UK, ${ }^{5}$ SLAC National Accelerator Laboratory, 2575 Sand Hill Road, Menlo Park, California 94025, USA, 'Lawrence Berkeley National Laboratory, 1 Cyclotron Road, California 94720, USA, ${ }^{7}$ Department of Physics and Photon Science, Gwangju Institute of Science and Technology, Gwangju, 500-712, Korea, ${ }^{8}$ Center for Relativistic Laser Science, Institute for Basic Science (IBS), Gwangiu, 500-712, Korea, 'Lawrence Livermore National Laboratory, 7000 East Avenue, Livermore, California 94550, USA, ${ }^{10} \mathrm{Helmholtz}$ Institute Jena, Fröbelstieg 3, 07743 Jena, Germany, ${ }^{1}$ Sorbonne Universités, Pierre et Marie Curie, UPMC, UMR 7605, LULI, case 128, 4 Place Jussieu, F-75252 Paris Cedex 05, France, ${ }^{12}$ Deutsches Elektronen-Synchrotron DESY, Notkestr. 85, 22607 Hamburg, Germany, ${ }^{13}$ Department of Astronomy and Astrophysics, University of Chicago, 5640 S. Ellis Ave, Chicago, IL 60637, USA, ${ }^{14}$ School of Mathematics and Physics, Queens University Belfast, Belfast BT7 1NN, United Kingdom, ${ }^{15}$ School of Physics and Astronomy, University of Edinburgh, Edinburgh EH9 3JZ, UK, ${ }^{16} \mathrm{GSI}$ Helmholtzzentrum für Schwerionenforschung GmbH, Planckstr. 1, 64291 Darmstadt, Germany, ${ }^{17}$ Institut für Physik, Universität Rostock, 18051 Rostock, Germany, ${ }^{18}$ Max-Planck-Institut für Physik Komplexer Systeme, 01187 Dresden, Germany, ${ }^{19}$ Institute of Optics and Quantum Electronics, Friedrich-Schiller-Universität Jena, Max-Wien-Platz 1, 07743 Jena, Germany, ${ }^{20}$ European XFEL GmbH, Albert-Einstein-Ring 19, 22761 Hamburg, Germany.
\end{abstract}

Here, we report results of an experiment creating a transient, highly correlated carbon state using a combination of optical and $x$-ray lasers. Scattered $x$-rays reveal a highly ordered state with an electrostatic energy significantly exceeding the thermal energy of the ions. Strong Coulomb forces are predicted to induce nucleation into a crystalline ion structure within a few picoseconds. However, we observe no evidence of such phase transition after several tens of picoseconds but strong indications for an over-correlated fluid state. The experiment suggests a much slower nucleation and points to an intermediate glassy state where the ions are frozen close to their original positions in the fluid.

\footnotetext{
(2) arbon is known to have many phases with extraordinary properties including the highest melting temperature of any material. Yet, the high-pressure transitions into phases with temperature above 5,000 K and densities above $1 \mathrm{~g} / \mathrm{cm}^{3}$ are poorly understood ${ }^{1,2}$. Carbon is expected to form a chemically inert phase under such extreme conditions, and exists in liquid form in the interiors of giant planets, such as Neptune, Uranus, and carbon-rich extrasolar planets ${ }^{3,4}$. The high-pressure phases of carbon also determine the state and evolution of more compact objects like white dwarf and neutron stars ${ }^{5,6}$.

Strongly driven systems far from equilibrium offer a very direct way of investigating the microscopic dynamics, inter-particle interactions and phase changes. High-pressure melting ${ }^{7}$, transient changes of optical properties ${ }^{8,9}$ and the relaxation path towards equilibrium ${ }^{10,11}$ are examples with high importance when studying materials with high energy density. Indeed, such states that naturally occur in large astrophysical bodies can only be created in the laboratory by depositing a large amount of energy into a sample and driving it far from equilibrium. However, the study of such warm dense matter (WDM) states poses severe challenges in both modeling and experimental diagnostics. Their temperature is too high for solid state theories to be applicable, but at the same time WDM is too cold for plasma perturbation techniques to converge since the thermal energy is only a fraction of the Coulomb energy between the particles ${ }^{12}$. Accessing WDM states experimentally has also remained rather challenging due to the inability to directly probe stellar matter and, in the laboratory, due to the difficulty in achieving well-diagnosed off-Hugoniot states created by large lasers and pulsed-power machines ${ }^{13}$. While some experiments have produced
} 
a transient liquid state of carbon through isochoric heating ${ }^{7}$, only limited structural and dynamical information could be obtained.

To overcome these limitations, we used here two nearly collinear laser beams to create a unique dense matter regime in an experiment performed at the Linac Coherent Light Source ${ }^{14}$. First we illuminated a $1 \mu \mathrm{m}$ thin graphite foil with an optical laser (see Figure 1 for details of the experimental setup). The laser was focused with a relatively large focal spot of $80 \mu \mathrm{m}$ diameter and a moderate intensity of $\sim 10^{15} \mathrm{~W} / \mathrm{cm}^{2}$. The corresponding ablation pressure of $\sim 30 \mathrm{Mbar}^{15}$ launches a shock wave through the carbon sample. The shock compresses the sample to densities of $\rho \sim 2.5-5 \mathrm{~g} / \mathrm{cm}^{3}$ and heats it to temperatures $T \sim 5,000-10,000 \mathrm{~K}$, inducing the carbon to melt (see also Ref. [2] for the high pressure phase diagram of carbon). Figure 2 shows an example for the density and temperature profiles after 40 ps as obtained from modelling the radiation driven hydrodynamics. After a time of $t \sim 40-50 \mathrm{ps}$, the shock reaches the opposite side of the foil and breaks out. This long wavelength, optical laser also produces a large number of nonthermal electrons having a highenergy tail with a temperature of $\sim 7.5-14 \mathrm{keV}^{16}$. These electrons will significantly enhance the ionization degree of the carbon sample by electron impact.
After the illumination by the optical laser, the $\mathrm{x}$-ray beam from the free electron laser (FEL) interacts with the pre-formed liquid state at different delays. The high intensity and photon energy of the FEL, $\sim 10^{17} \mathrm{~W} / \mathrm{cm}^{2}$ and $E_{\mathrm{x}}=8 \mathrm{keV}$, leads to additional ionisation of the sample ${ }^{8,9}$. While the FEL intensity is only sufficient to photo-ionize about $0.1 \%$ of the ions in the probed volume directly, the energy of the ejected electron, being the difference between the photon energy and the K-shell binding energy of the ions, is large allowing for the creation of several secondary electrons contributing roughly $10 \%$ of the total ionization of the sample.

Photo and impact ionisation are enhanced by Auger recombination of core holes that are filled within a few femto-seconds ${ }^{17}$ and results in the ejection of an L-shell electron. Therefore, the ionization of the sample is expected to be far above the charge state of a system in thermodynamic equilibrium. Given that electrons with energy $\gtrsim 7.5 \mathrm{keV}$ will perform many ionization events during the FEL pulse $^{18}$ and have a range of less than $1 \mu \mathrm{m}$, most of the carbon sample in the probed region will consist of highly charged ions. The hot electrons also deposit additional energy into the background plasma, raising the electron temperature by a few $\mathrm{eV}$ in a time shorter than the duration of the FEL pulse. On the other hand, electron-ion

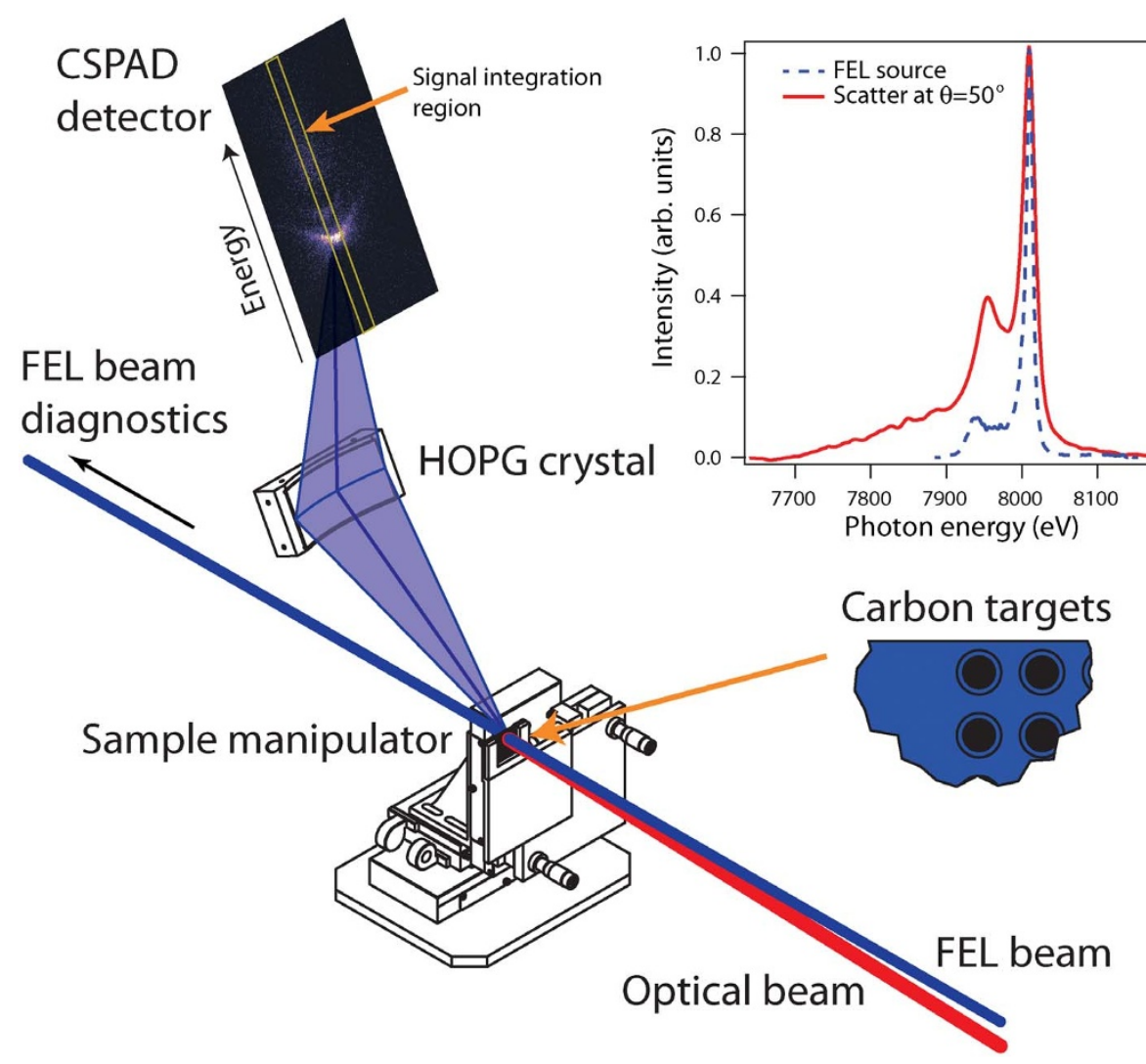

Figure 1 Experimental setup. This work was been performed at at the LCLS XPP instrument. A 300 fs long, $10 \mathrm{~mJ}$ Ti:Sapphire laser (operating at wavelength of $800 \mathrm{~nm}$ ) was focused onto a $1 \mu \mathrm{m}$ thick graphite foil, mounted on a rastering stage within a vacuum chamber, to a focal spot-size of $80 \mu \mathrm{m}$ diameter resulting in an intensity on target of $10^{15} \mathrm{~W} / \mathrm{cm}^{2}$. The shocked heated carbon foil was then illuminated by the FEL probe beam (operating at $8 \mathrm{keV}$, with $\sim 1 \mathrm{~mJ}$ energy in a $80 \mathrm{fs}$ long pulse) propagating nearly collinearly with the optical laser pulse. The $\mathrm{x}$-ray spot size was around $20 \mu \mathrm{m}$ diameter, focused using a series of beryllium refractive lenses. The inset shows the spectrum of the $\mathrm{x}$-ray beam as it reaches the sample, as well as the measured scattering spectrum at $\theta=50^{\circ}$. Temporal synchronization and spatial overlap of the optical and $\mathrm{x}$ ray beams was achieved through the use of optical damage shadowgraphy ${ }^{35}$. The scattered $\mathrm{x}$-rays are collected using a highly oriented pyrolytic graphite (HOPG) crystal spectrometer in von Hàmos geometry. Details of the crystal spectrometer calibration are given in Ref. [23]. Data is then recorded onto a high quantum efficiency, high repetition rate pixel-array detector (CSPAD). An example of the raw data recorded on the detector is shown in the figure. The diffracted photon energy increases from bottom to top in the image. From the raw image, the spectrum was obtained by integrating along the non-dispersive direction, but only in a narrow central strip, as indicated in the figure. This is to minimize spectral broadening associated to crystal aberrations, as discussed in Ref. [23]. The spectrometer, including detector, was mounted to a six-axis robotic arm, enabling the angle at which the scattered radiation was measured to be varied during the experiment $\left(\theta=20^{\circ}, 35^{\circ}, 50^{\circ}, 130^{\circ}\right)$. The HOPG crystal was large enough to collect rays from $\pm 5^{\circ}$ of the nominal scattering angle. The polarization of the $\mathrm{x}$-ray beam was at $90^{\circ}$ to the angle of scatter, maximizing the scattering efficiency and obviating any polarization effects on the scattered radiation. 
a)

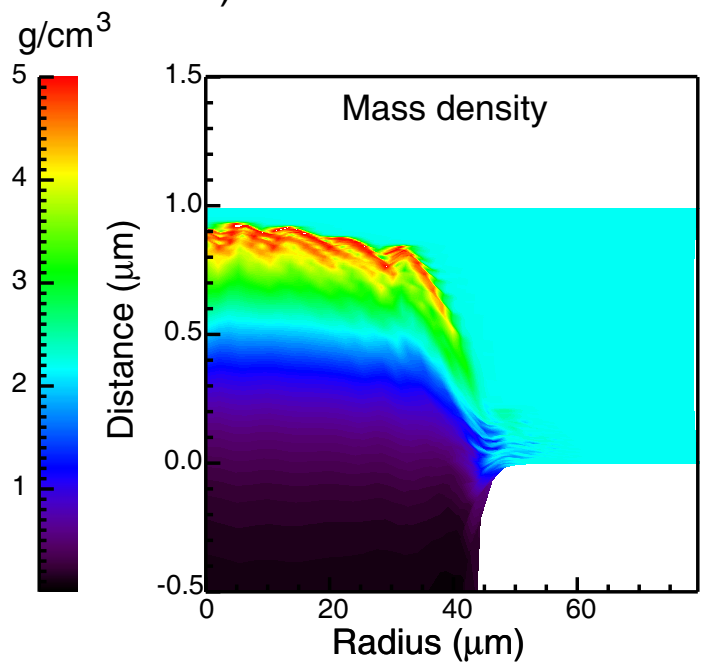

b)

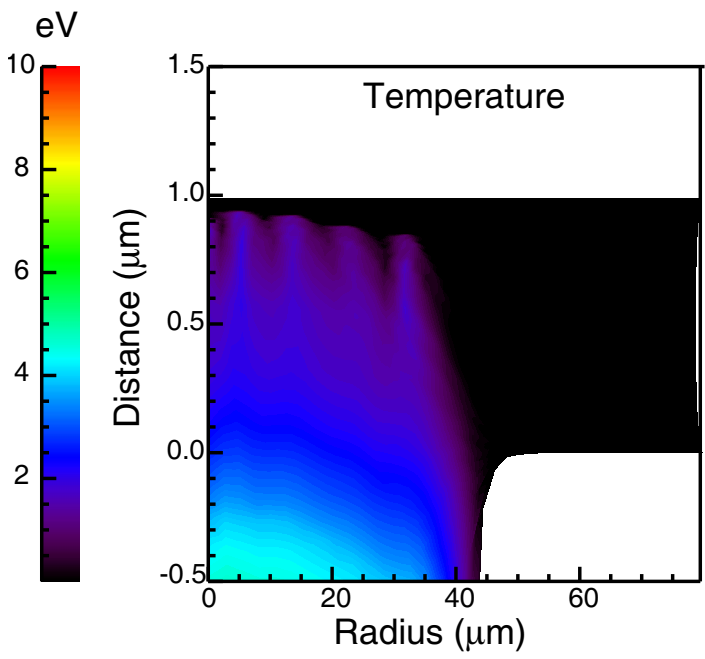

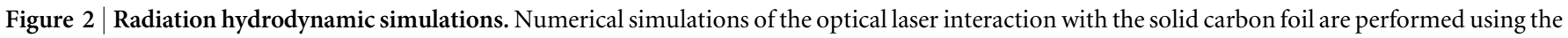

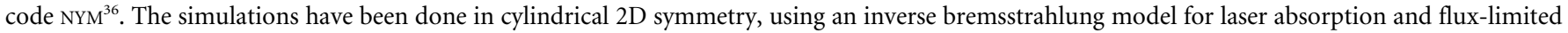

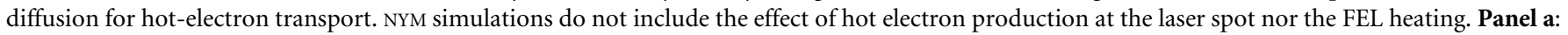
contour plot of the mass density at $t=40 \mathrm{ps}$; Panel b: electron temperature at $t=40$ ps after optical laser arrival time.

equilibration occurs on much longer times ${ }^{10}$ - typically hundreds of picoseconds - and thus the carbon ions remain at the temperature created by the shockwave, that is, $T_{i} \sim 5,000-10,000 \mathrm{~K}$.

\section{Results}

The FEL beam delivers enough photons that a sufficient number are scattered by the electrons in the plasma and can be used as diagnostics $^{19,20}$. The power of the scattered photons is given by $d P / P_{0}=$ $n_{i} \ell \sigma_{T} S_{\text {tot }}(k) d \Omega$, where $P_{0}$ is the incident power of x-rays, $n_{i}$ is the ion density, $\sigma_{T}$ is the Thomson cross section, $d \Omega$ is the acceptance solid angle of the analyzer crystal (see Figure 1 ), and $S_{\text {tot }}(k)$ is the total electron structure factor. $S_{\text {tot }}(k)$ reflects the spatial arrangements of the electrons in the sample and, thus, relates the scattered power spectrum to the material properties. It is probed at a wavenumber of $k=(4 \pi / \lambda) \sin (\theta / 2)$, where $\lambda=1.5 \AA(8 \mathrm{keV})$ is the x-ray wavelength and $\theta$ is the scattering angle.

As plotted in the inset of Figure 1, the scattered radiation shows two distinct features: i) an elastic (or nearly elastic) peak at the original wavelength, and ii) a frequency-shifted feature. The first feature is the result of scattering by electrons that kinematically follow the ions ${ }^{21,22}$, which in WDM can be bound or form the screening cloud around the ions. The second feature, which is smaller in intensity but still contributing a significant amount of the total signal (see Figure 1), results from inelastic scattering off free electrons and Compton scattering from core electrons which causes photo-ionization. We used a highly oriented pyrolitic graphite (HOPG) crystal analyzer ${ }^{23}$ in order to separate the elastic from the inelastic scattering. The measured spectra were furthermore deconvolved by taking into account the measured spectral profile of the FEL source. Any diffuse scattering arising from the polycrystalline structure of the cold (unshocked) region of sample is removed by subtracting the measured scattering signal without optical laser heating (i.e., FEL only) weighted by the mass fraction of unshocked material. At any given time the latter is estimated from the results of a radiation-hydrodynamics simulation (see Figure 2). The cold scattering signal is plotted in Figure 3a. The cold graphite structure is hexagonal close-packed (hcp). The (012) diffraction line contributes to the measured peak at $3.4 \AA^{-1}\left(\theta=50^{\circ}\right)$.

Information about the ion structure in the sample is contained in the coherent nearly elastic scattering peak. The corresponding part of the electron structure factor is given by $|f(k)+q(k)|^{2} S_{\text {ii }}(k)$, where $f(k)$ refers to the atomic form factor of bound electrons and $q(k)$ to the screening cloud of electrons around an ion $^{21}$. In our experiment, the sum of $f(k)$ and $q(k)$ is only weakly dependent on the details of the plasma conditions as we have $k \lambda_{T F} \gtrsim 1\left(\lambda_{T F}\right.$ is the Thomas-Fermi screening length) and $k a_{1 s} \lesssim 1$ ( $a_{1 s}$ is the radius of the 1 s electrons in carbon). Thus, $q(k)$ is very small and $f(k) \sim f(0)$ is almost constant. The main modulation of the elastic scattering signal results from the ion-ion structure factor, $S_{\text {ii }}(k)$, which describes the ion positions in Fourier space. To account for the detector response and transmission efficiency of the $\mathrm{x}$-ray collection optics, we have used as the normalization condition that for $k \gtrsim 7 \AA^{-1}$ the structure factor should tend to unity. This diffraction technique, recently implemented to study correlations in dense aluminum ${ }^{24}$, is different from the commonly used angle-resolved, frequency-integrated methods. The latter are, however, unable to provide a direct measurement of the ion structure if inelastic scattering is large, as shown in Figure 1.

Our results for the ion-ion structure factor extracted from the elastic scattering feature are shown in Figure 3b, with the error analysis presented in Figure 3c, for various delays between the optical laser and the $\mathrm{x}$-ray probe. The measured rise of the structure factor at small $k$, as well as a finite value at large scattering angles is typical for a strongly coupled liquid. The presence of a high correlation peak indicates that the carbon ions exhibit a pronounced short-range order on the scale of $2 \pi / k_{0} \sim 2 \AA$ with $k_{0} \sim 3.4 \AA^{-1}$ being the position of the first peak in the structure factor for the conditions in our experiment. While the cold data also show a peak at the same position, the fact that the diffraction intensity is much stronger when the sample is heated, and the appearance of non zero scattering at $\sim 7.4$ $\AA^{-1}\left(\theta=130^{\circ}\right)$ both suggest a substantial change from the initial hcp carbon structure. This new order is a result of the strong Coulomb repulsion between the highly charged ions. Indeed, such a structure factor is indicative for systems where the Coulomb energy between the ions significantly exceeds their thermal excitation. The ratio of these two energies is usually expressed by the parameter $\Gamma=$ $Z^{2} e^{2} / 4 \pi \epsilon_{0} a k_{B} T \gg 1$ with $a=\left(3 / 4 \pi n_{i}\right)^{1 / 3}$ being the mean distance between ions (where $n_{i}$ is the ion density). Moreover, the total pressure of such systems, expressed as $p=p_{\text {id }}+p_{\text {ex }}$, is dominated by the excess pressure due to ion-ion repulsion and not by the ideal contribution, $p_{\text {id }}$, as in a gas or typical plasma ${ }^{25}$. The Coulomb forces between ions thus dominate the thermodynamics of the system. 
a)

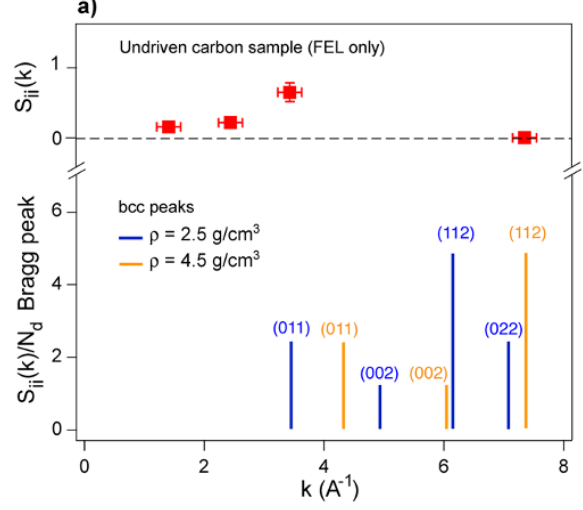

b)

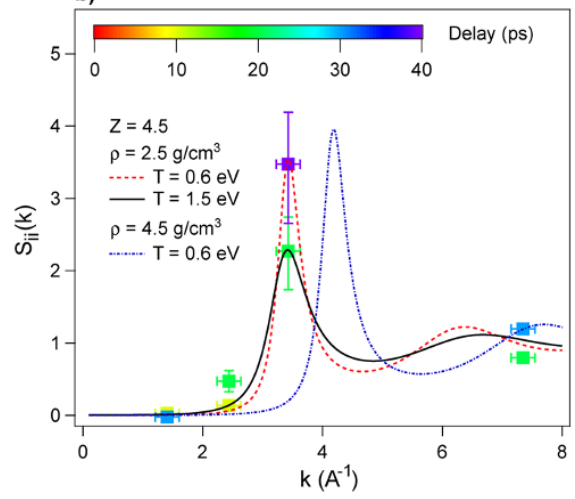

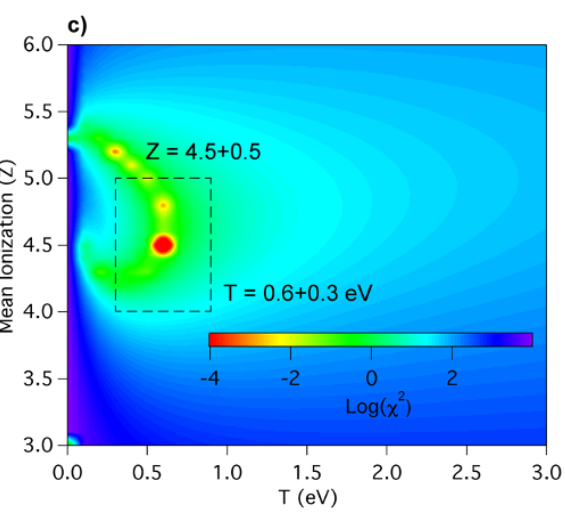

Figure 3 Structure factor of strongly coupled carbon. Panel a: scattering data from the un-shocked (cold) graphite. The position and relative intensity of the bcc lattice peaks, labelled according their Miller indices, for $\rho=2.5 \mathrm{~g} / \mathrm{cm}^{3}$ (blue lines) and $\rho=4.5 \mathrm{~g} / \mathrm{cm}^{3}$ (orange lines) is indicated in the figure. The Bragg peak intensity is estimated by assuming $\eta_{\Gamma}=0.1$. Panel b: data were collected at a repetition rate of approximately a shot per 10 seconds, allowing for the coherent scattering to be taken at various combinations of beam delays and measurement angle. Each data point consists of an average of about 50-60 individual shots. The FEL energy has been individually recorded for each shot, and then single spectra have been weighted by the corresponding energy in the FEL beam (with cold scattering removed). The experimental values for $S_{\mathrm{ii}}(k)$ were derived from the total elastic scattering by assuming $Z=4.5 \pm 0.5$. The uncertainty in $Z$ determines the vertical error bars. The standard deviation in signal intensity from shot-to-shot variations in a data set is within the reported errors. Horizontal error bar are related to the finite acceptance angle of the spectrometer. The calculated curves are results from a fluid model for charged ions embedded in a polarisable background of electrons for a density of $\rho=2.5 \mathrm{~g} / \mathrm{cm}^{3}$ and $\rho=4.5 \mathrm{~g} / \mathrm{cm}^{3}$, an ion charge state of $Z=4.5$, and two different temperatures ( $1 \mathrm{eV}$ equals $11,600 \mathrm{~K})$. Panel c: plot of $\chi^{2}=\left|S_{\mathrm{ii}}^{\exp }-S_{\mathrm{ii}}^{\mathrm{MSA}}\right|^{2}$ versus $T$ and $Z$, where $S_{\mathrm{ii}}^{\exp }=I^{\exp } /|f(k)+q(k)|^{2}$ is the structure factor derived from the measured data $\left(I^{\exp }\right)$ and $S_{\mathrm{ii}}^{\mathrm{MSA}}$ is the calculation for $\rho=2.5 \mathrm{~g} / \mathrm{cm}^{3}, \theta=50^{\circ}$ and $40 \mathrm{ps}$ delay.

In the case of a plasma in thermodynamic equilibrium, density functional theory coupled to molecular dynamics (DFT-MD) simulations represent an $a b$ initio method to calculate the ion-ion structure factor ${ }^{26}$. The result of such simulations is shown in Figure 4. We notice that for the conditions of our experiment, DFT-MD gives an ion-ion structure factor which is relatively flat in the region of $k \gtrsim 3 \AA^{-1}$ indicating a moderately coupled system only. By comparing the DFT-MD results with the experimental data (Figure 3 ), we see that the agreement is rather poor, particularly in the region of the first peak of the structure factor. This is due to the fact that an equilibrium calculation only predicts a moderate ionization for carbon $(Z \sim 2)$, whereas in our experiment, additional ionization is driven by suprathermal electrons while keeping the initial density and ion temperature almost unaffected. Hence, the plasma state created in our experiment is better described in terms of positively charged ions with a much higher charge state embedded in a polarisable background of electrons ${ }^{27}$. This situation can often be described by the mean spherical approximation (MSA) making the problem of the ion structure analytically solvable. The best fit with the experimental data is obtained for an ion charge state of $Z=4.5$ (Figure 3). The calculations shown in Figure 4 clearly indicate that as the ionization increases there is the emergence of a strong correlation peak. The position of the peak depends on the density, thus the crystal analyzer placed at $\sim 3.4 \AA^{-1}\left(\theta=50^{\circ}\right)$ primarily selects scattering coming from region in the sample where $\rho=2.5 \mathrm{~g} / \mathrm{cm}^{3}$. On the other hand, different densities are equally weighted in the scattering signal, when the crystal analyzer selects $\sim 7.4 \AA^{-1}$ at a scattering angle of $\theta=130^{\circ}$.

\section{Discussion}

The highly charged ions and the resulting strong Coulomb forces between them should have further implications for the dynamics of the system. As shown in Fig. 3b, the structure factor exhibits a strong correlation peak with a maximum of $S_{i i}\left(k_{0}\right) \sim 3.5$ at $t>30$ ps. According to the Hansen-Verlet criterion ${ }^{28}$, a first correlation peak with $S_{i i}\left(k_{0}\right)>2.85$ indicates a phase transition into a solid with a well-defined lattice structure. The measured values in our experiment clearly fulfill this criterion even if one considers possible errors in determining the exact peak value (see Figure 3 ). Thus, we find the situation that we have measured a fluid structure that itself indicates that a phase transition into a solid, that is a Coulomb crystal, should occur at these conditions.

Recent models predict that crystalline structures consisting of a large number of ions should already have formed in the time span between the optical laser and FEL probe ${ }^{29}$. Numerical simulations give nucleation times of $\tau \approx r_{c} a\left(k_{B} T / M\right)^{-1 / 2} \sim$ a few picoseconds ${ }^{30,31}$, where $M$ the ion mass and $r_{c} \sim 10-50$ the critical radius for nucleation (in units of $a$ ). Since $\tau$ is shorter than the characteristic

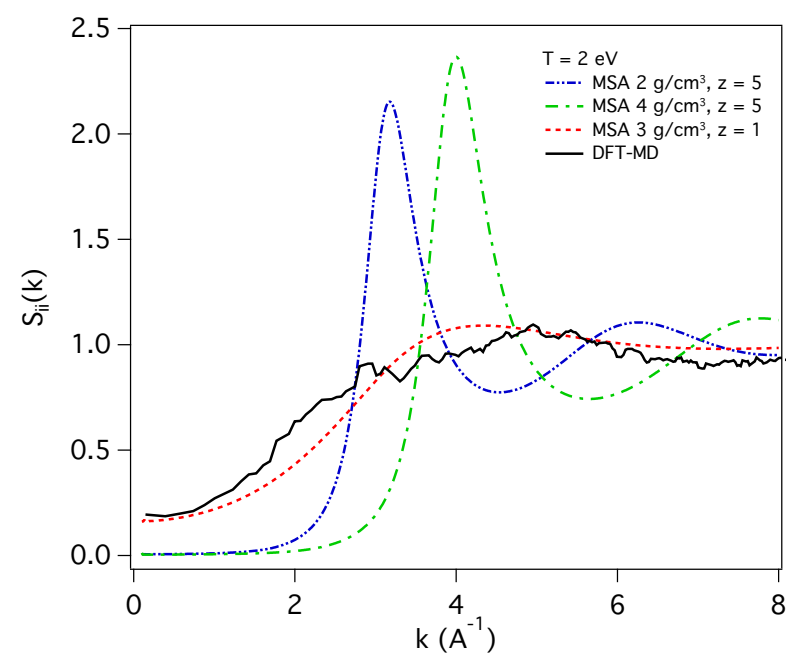

Figure $4 \mid$ Ion-ion structure factor calculations. A comparison between DFT-MD and MSA predictions for the ion-ion structure factor. In density functional simulations both electrons and ions are handled as elementary particles. Properties of the electrons are calculated via density functional theory using a Mermin functional that accounts for temperature effects within the electron subsystem in a statistical sense. The ions instead are treated by classical molecular dynamics simulations. This is possible because the dynamics of ions and electrons is effectively decoupled with the Born-Oppenheimer approximation. By taking snapshots of the ions positions, the ion-ion structure factor can be thus calculated. Our ab initio calculations are performed with $\rho=3 \mathrm{~g} / \mathrm{cm}^{3}$ and $T=30,000 \mathrm{~K}$. MSA calculations ${ }^{27}$ instead use the ionization state $(Z)$ as an additional input. 
hydrodynamic times (typically tens of picoseconds), the dense plasma should have had enough time to evolve into a crystal, a process known as Wigner crystallization ${ }^{12}$. However, our measurements show no indication of a crystalline structure which points to a state where the ions are locked to a fluid-like structure by strong forces. Given the pump-probe difference used, this state exists much longer than typical nucleation models predict.

In order to test the possibility of a crystalline state, we infer $\Gamma \gtrsim 600$ at peak compression with $\rho \gtrsim 4.5 \mathrm{~g} / \mathrm{cm}^{3}$ and $T \sim$ $5,000 \mathrm{~K}$ from the hydrodynamics simulations (see Figure 2). At such large values of the coupling parameter, a system of ions embedded into a screening background of electrons forms a crystalline state with a body centered cubic (bcc) lattice ${ }^{12}$, as the phase transition is found for $\Gamma_{c} \sim 400^{32}$. Thus, the highly compressed states in the sample are clearly at conditions where we expect a solid. The ratio of the scattering power between the solid and liquid phases in the $(h k l)$ direction is $d P_{\text {solid }} / d P_{\text {liquid }} \sim \eta_{\Gamma}\left(N_{d} / 2\right) m_{h k l}\left|F_{h k l}\right|^{2}$. Here $N_{d} \gg 1$ is the number of ions in each solid crystallite that has nucleated, $\eta_{\Gamma}$ is the fraction of the sample where $\Gamma \gtrsim 400, m_{h k l}$ and $F_{h k l}$ are the multiplicity and the structure factor, respectively, for Bragg reflections along the plane of Miller indices $(h k l)$. The hydrodynamics simulations (see Figure 2) indicate $\eta_{\Gamma} \approx 8 \%-10 \%$ (for sample densities $\rho=4.5 \pm 0.25 \mathrm{~g} / \mathrm{cm}^{3}$ ). Therefore, scattering from this newly formed bcc structure should be visible in the data.

In Figure 3, we have indicated the positions of the Bragg scattering peaks for the bcc lattice. For the (112) peak at $4.5 \mathrm{~g} / \mathrm{cm}^{3}$, we expect a large ratio of the scatter from the solid to the fluid. However, the observed scattering at the (112) position remains comparable to the signal measured for the (011) plane at $2.5 \mathrm{~g} / \mathrm{cm}^{3}$. This indicates that, in contradiction to the nucleation models, any significant crystallization has not occurred at the probe time.

It has been suggested theoretically that the transition to a solid is suppressed after rapid changes of the interaction potential between the particles leading to a metastable amorphous or glassy state in Coulomb systems ${ }^{31}$. One reason for such a behaviour is that the transition to a strongly coupled fluid with coordination numbers of 3 to 4 is much easier to achieve than the one to a bcc lattice with a coordination number of 8 . Since we have measured a fluid-like structure for conditions for which a bcc lattice should exist, we conclude that we have found compelling indications for such a Coulomb glass. Both the Hansen-Verlet criterion ${ }^{28}$, which is independent of the specific form of the interactions but based on the measured height of the first correlation peak, as well as our analysis using the radiation-hydrodynamics simulations support this finding. Although we can rule out the known solid phases to exist for the conditions probed, the determination of the full structure in the proposed glassy state will require additional experiments revealing the full $k$-dependence of the structure factor, as in conventional angle-resolved $x$-ray diffraction.

The plasma state explored in this experiment $\left(\rho \gtrsim 2.5 \mathrm{~g} / \mathrm{cm}^{3}\right.$, and $T \lesssim 1 \mathrm{eV}$ ) lies within the parameter range of the envelope of white dwarfs with lower luminosity ${ }^{33}$. Due to the enhanced ionization state, it is also representative of the strongly coupled plasma found in their deeper interior, as well as in neutron star matter. The existence of a glassy state in white dwarfs would affect their thermal conductivity. While the duration of the metastable glassy state is most likely not long enough compared to the characteristic cooling time of white dwarf stars (which is several billion years), it may nevertheless be comparable to the cooling times of neutron stars ${ }^{34}$, thus even affecting their overall luminosity.

1. Correa, A. A., Bonev, S. A. \& Galli, G. Carbon under extreme conditions: Phase boundaries and electronic properties from first-principles theory. Proc. Natl. Acad. Sci. 103, 1204-1208 (2006).

2. Knudson, M., Desjarlais, M. \& Dolan, D. Shock-wave exploration of the highpressure phases of carbon. Science 322, 1822-1825 (2008).
3. Guillot, T. Interiors of giant planets inside and outside the solar system. Science 286, 72-77 (1999).

4. Benedetti, L. R. et al. Dissociation of $\mathrm{CH} 4$ at high pressures and temperatures: diamond formation in giant planet interiors? Science 286, 100-102 (1999).

5. Van Horn, H. Dense astrophysical plasmas. Science 252, 384-389 (1991).

6. Dufour, P., Liebert, J., Fontaine, G. \& Behara, N. White dwarf stars with carbon atmospheres. Nature 450, 522-524 (2007).

7. Pelka, A. et al. Ultrafast melting of carbon induced by intense proton beams. Phys. Rev. Lett. 105, 265701 (2010).

8. Nagler, B. et al. Turning solid aluminium transparent by intense soft $\mathrm{x}$-ray photoionization. Nature Phys. 5, 693-696 (2009).

9. Vinko, S. et al. Creation and diagnosis of a solid-density plasma with an $\mathrm{x}$-ray freeelectron laser. Nature 482, 59-62 (2012).

10. White, T. et al. Observation of inhibited electron-ion coupling in strongly heated graphite. Sci. Rep. 2, 889 (2012).

11. Chapman, D. \& Gericke, D. Analysis of x-ray Thomson scattering for nonequilibrium plasmas. Phys. Rev. Lett. 107, 165004 (2011).

12. Ichimaru, S. Strongly coupled plasmas: high-density classical plasmas and degenerate electron liquids. Rev. Mod. Phys. 54, 1017 (1982).

13. Remington, B. A., Drake, R. P. \& Ryutov, D. D. Experimental astrophysics with high power lasers and z pinches. Rev. Mod. Phys. 78, 755 (2006).

14. Emma, P. et al. First lasing and operation of an Angstrom-wavelength freeelectron laser. Nature Photonics 4, 641-647 (2010).

15. Drake, R. P. High energy density physics (Springer, 2006).

16. Reich, C., Gibbon, P., Uschmann, I. \& Föster, E. Yield optimization and time structure of femtosecond laser plasma K $\alpha$ sources. Phys. Rev. Lett. 84, 4846-4849 (2000).

17. Sette, F. et al. Lifetime and screening of the $\mathrm{C} 1 \mathrm{~s}$ photoemission in graphite. Phys. Rev. B 41, 9766 (1990).

18. Salzmann, D. Atomic physics in hot plasmas, vol. 97 (Oxford University Press, USA, 1998).

19. Garcia Saiz, E. et al. Probing warm dense lithium by inelastic x-ray scattering Nature Phys. 4, 940-944 (2008).

20. Glenzer, S. H. \& Redmer, R. X-ray Thomson scattering in high energy density plasmas. Rev. Mod. Phys. 81, 1625 (2009).

21. Chihara, J. Interaction of photons with plasmas and liquid metalsphotoabsorption and scattering. J. Phys.: Condens. Matter 12, 231 (1999).

22. Gregori, G., Glenzer, S., Rozmus, W., Lee, R. \& Landen, O. Theoretical model of $\mathrm{x}$ ray scattering as a dense matter probe. Phys. Rev. E 67, 026412 (2003).

23. Zastrau, U. et al. Focal aberrations of large-aperture HOPG von-Hàmos X-ray spectrometers. J. Inst. 7, P09015 (2012).

24. Ma, T. et al. X-ray scattering measurements of strong ion-ion correlations in shock-compressed aluminum. Phys. Rev. Lett. 110, 065001 (2013).

25. Galam, S. \& Hansen, J.-P. Statistical mechanics of dense ionized matter. vi. electron screening corrections to the thermodynamic properties of the onecomponent plasma. Phys. Rev. A 14, 816 (1976).

26. Wünsch, K., Vorberger, J. \& Gericke, D. Ion structure in warm dense matter: Benchmarking solutions of hypernetted-chain equations by first-principle simulations. Phys. Rev. E 79, 010201(R) (2009).

27. Palmer, R. \& Weeks, J. Exact solution of the mean spherical model for charged hard spheres in a uniform neutralizing background. J. Chem. Phys. 58, 4171-4174 (1973).

28. Hansen, J.-P. \& Verlet, L. Phase transitions of the Lennard-Jones system. Phys. Rev. 184, 151-161 (1969).

29. Cooper, R. \& Bildsten, L. Classical nucleation theory of the one-component plasma. Phys. Rev. E 77, 056405 (2008).

30. Daligault, J. Crystal nucleation in the one-component plasma. Phys. Rev. E 73, 056407 (2006).

31. Ichimaru, S., Iyetomi, H., Mitake, S. \& Itoh, N. Amorphous glassy plasma in dense stellar matter. Astrophys. J. 265, L83-L86 (1983).

32. Hamaguchi, S., Farouki, R. \& Dubin, D. Triple point of Yukawa systems. Phys. Rev. E 56, 4761-4682 (1997).

33. Fontaine, G. \& Van Horn, H. Convective white-dwarf envelope model grids for $\mathrm{H}$ , He-, and C-rich compositions. Astrophys. J. Suppl. 31, 467-487 (1976).

34. Van Riper, K. \& Lamb, D. Neutron star evolution and results from the Einstein $\mathrm{x}$ ray observatory. Astrophys. J. 244, L13-L17 (1981).

35. Harmand, M. et al. Plasma switch as a temporal overlap tool for pump-probe experiments at FEL facilities. J. Inst. 7, P08007 (2012).

36. Roberts, P. D., Rose, S. J., Thompson, P. C. \& Wright, R. J. The stability of multiple-shell ICF targets. J. Phys. D 13, 1957 (1980).

\section{Acknowledgments}

This work was supported by EPSRC grants EP/G007187/1, EP/G007462/1, and EP/ D062837. Travel to LCLS was supported through EPSRC grant EP/I018484. In the United Kingdom, additional support from AWE and the Science and Technology Facilities Council is acknowledged. In Germany, this work was supported by the the DFG, the German Ministry for Education and Research via FSP-301, and the Volkswagen Foundation. B.I.C. was supported by the NRF of Korea (grant number: 2013R1A1A1007084) and by the TBP project of GIST. Portions of this research were carried out on the XPP instrument at the 
LCLS, a division of SLAC National Accelerator Laboratory and an Office of Science user facility operated by Stanford University for the US Department of Energy.

\section{Author contributions}

G.G. conceived this project. The paper was written by C.R.D.B., D.O.G., D.Q.L., J.V., T.T. and G.G. The experimental work was performed by C.R.D.B., M.C., B.I.C., T.D., K.E., D.F., E.G., M.H., P.H., N.L.K., H.J.L., H.L., M.M., A.M., C.D.M., B.N., P.N., D.R., F.B.R., S.T., S.M.V., S.W., U.Z., D.Z., T.T. and G.G. Data analysis and supporting numerical simulations were done by C.R.D.B., D.O.G., K.-U.P., R.R., P.S., J.V., T.G.W. and K.W. Additional theoretical support was provided by C.F., E.F., S.H.G. and R.W.L.

\section{Additional information}

Competing financial interests: The authors declare no competing financial interests.

How to cite this article: Brown, C.R.D. et al. Evidence for a glassy state in strongly driven carbon. Sci. Rep. 4, 5214; DOI:10.1038/srep05214 (2014).

(c) (i) $\odot$ This work is licensed under a Creative Commons Attribution-NonCommercialNoDerivs 3.0 Unported License. The images in this article are included in the article's Creative Commons license, unless indicated otherwise in the image credit; if the image is not included under the Creative Commons license, users will need to obtain permission from the license holder in order to reproduce the image. To view a copy of this license, visit http://creativecommons.org/licenses/by-nc-nd/3.0/ 